\title{
Improved dynamic programs for some batching problems involving the maximum lateness criterion
}

\author{
A P M Wagelmans \\ Econometric Institute \\ Erasmus University Rotterdam \\ PO Box 1738, 3000 DR Rotterdam \\ The Netherlands \\ A E Gerodimos \\ Centre for Quantitative Finance \\ Imperial College \\ Exhibition Road \\ London, SW7 2BX \\ United Kingdom
}

April 27, 1999

\begin{abstract}
We study four scheduling problems involving the maximum lateness criterion and an element of batching. For all the problems that we examine, algorithms appear in the literature that consist of a sorting step to determine an optimal job sequence, followed by a dynamic programming step that determines the optimal batches. In each case, the dynamic program is based on a backward recursion of which a straightforward implementation requires $O\left(n^{2}\right)$ time, where $n$ is the number of jobs. We present improved implementations of these dynamic programs that are based on monotonicity properties of the objective expressed as a function of the total processing time of the first batch. These properties and the use of efficient data structures enable optimal solutions to be found for each of the four problems in $O(n \log n)$ time; in two cases, the batching step is actually performed in linear time and the overall complexity is determined by the sorting step.
\end{abstract}




\section{Introduction}

The early 1990's saw the emergence of powerful techniques that reduced the time requirement of dynamic programming algorithms for the classic economic lot sizing (ELS) problem $[8,14,1]$. It was subsequently realized that certain scheduling problems involving the sum of completion times objective and an element of batching exhibited structural properties that made them amenable to more efficient dynamic programming solutions. In some cases $[7,3]$, the improved schemes were problem-specific; in other cases $[6,10]$, the dynamic programming recursion could be written in a form that allowed the application of the geometric techniques of Van Hoesel et al. [13], which are a generalization of the technique used in [14]. The typical complexity improvement was from $O\left(n^{2}\right)$ to $O(n \log n)$, where $n$ is the number of jobs. A question that arises naturally is whether similar improvements can be achieved in solving the maximum lateness counterparts of these batching problems since, in a standard implementation, the respective dynamic programs have also quadratic time requirements. This paper provides an affirmative answer to this question. We study four such batching problems and provide implementations of dynamic programming with a time requirement that is either linear or $O(n \log n)$. Since the batching problems are solved after an initial sorting step, our results imply $O(n \log n)$ algorithms for the four maximum lateness problems.

The remainder of this paper is organized as follows. In Section 2 we sketch our approach with particular focus on a subproblem that we encounter frequently when solving the four batching problems. Subsequently, we list the problems in order of relative complexity, both in terms of the improved running time and the difficulty of obtaining this improvement. Specifically, Section 3 deals with the problem of batching jobs of a single type under batch availability. A problem in which jobs are processed by a batching machine is the subject of Section 4. In Section 5 we give an improved algorithm for batching customized two-operation jobs on a single machine under batch availability and we indicate how a similar approach can be adopted in the case of item availability. Finally, some concluding remarks are given in Section 6 .

\section{Preliminaries}

In general, solving scheduling problems with a batching element involves taking the appropriate batching and sequencing decisions. For the problems 
that we examine in this paper, these two aspects can be decoupled. In fact, for three of our problems there is an optimal schedule in which jobs complete according to the earliest due date (EDD) rule, whereas for the problem studied in Section 4 the shortest processing time (SPT) rule is optimal. In any case, the sorting step imposes a lower bound of $O(n \log n)$ on the overall complexity of any algorithm. For two of the problems examined here, improving the efficiency of the dynamic programming step results in the sorting step being the overall bottleneck.

Although it is difficult to provide a description of a procedure that would be general enough to be applied to all the problems tackled in this paper, we now sketch some common elements of our approach; the implementation details and some special data structures deployed are covered in the subsequent sections.

Our starting point is always a backward recursion dynamic program with batch insertion [11]: the optimal schedule is built by inserting entire batches of jobs (or operations) at the start of previously obtained schedules. The recursions are thus of the general form:

$$
G_{k}=\min _{k<l \leq n+1}\left\{\max \left\{P_{k, l}+G_{l}, L_{k, l}\right\}\right\},
$$

where $G_{k}$ is the minimum lateness of schedules including jobs $k, k+1, \ldots, n$, whereas $P_{k, l}$ and $L_{k, l}$ denote the total processing time and overall lateness of the inserted batch, which consists of the jobs $k, k+1, \ldots, l-1$. In other words, $l$ is the index of the first job in the second batch.

Our first step is to observe that the index set $\{k+1, \ldots, n+1\}$ can be divided into two mutually exclusive index sets $I_{k}^{1}$ and $I_{k}^{2}$ so that the maximum in (1) is given by either the first or the second term, respectively. In view of that, (1) can be re-written as

$$
\left.G_{k}=\min \left\{\min _{l \in I_{k}^{1}}\left\{P_{k, l}+G_{l}\right\}, \min _{l \in I_{k}^{2}} L_{k, l}\right\}\right\},
$$

For reasons that will become clearer in the subsequent sections, the solution to the second minimization problem in (2) is obtained by retrieving the minimal index $l^{*}$ from $I_{k}^{2}$. This leaves us with the following tasks:

a) maintain/update the index sets $I_{k}^{1}$ and $I_{k}^{2}$ efficiently,

b) solve the first minimization problem and, where applicable, a second optimization problem that arises when calculating $L_{k, l^{*}}$.

With respect to the first task, observe that $I_{k}^{1}$ and $I_{k}^{2}$ are not necessarily contiguous. In fact, as we show in the subsequent sections, the satisfaction 
of this additional condition by some problems leads to linear-time implementations; where this is not the case, updating these index sets is 'costly' and the complexity of the dynamic program becomes $O(n \log n)$.

As for the second task, both the first minimization problem and the non-trivial variants of the second optimization problem possess a key property that enables a solution to be found in time which is overall linear. Specifically, the idea is to transform all such problems into a problem of the following type:

Problem (P) Determine the minima $m_{k}, k=1,2, \ldots, n$, defined as

$$
m_{k}=\min _{k<l \leq u_{k}} f_{l}
$$

where $u_{k} \in\{k, k+2, \ldots, n\}$ for all $k=1,2, \ldots, n\left(m_{k}=\infty\right.$ if $\left.u_{k}=k\right)$ and the following conditions hold:
a. $u_{k} \leq u_{k+1}$ for all $k=1,2, \ldots, n-1$
b. $u_{n}=n$
c. $u_{k}$ is known once $m_{k+1}$ is known, $k=1,2, \ldots, n-1$
d. $f_{k}$ is known once $m_{k}$ is known, $k=1,2, \ldots, n$

Conditions (c) and (d) suggest that the values $m_{k}$ can only be calculated in order of decreasing index $k$. A straightforward way to solve problem $(\mathrm{P})$ requires $O\left(n^{2}\right)$ time. We show, however, that a linear time bound is possible.

Consider, for an arbitrary $k \in\{1,2 \ldots, n\}$ with $u_{k}>k$, the values $f_{l}, l=k+1, k+2, \ldots, u_{k}$. Let $t(1), t(2), \ldots, t(r)$ be the unique subsequence of $k+1, k+2, \ldots, u_{k}$ which has the following properties:

1. $t(1)=k+1$,

2. $t(i+1)$ is the smallest index in $\left\{t(i)+1, t(i)+2, \ldots, u_{k}\right\}$ such that $f_{t(i+1)}<f_{t(i)}, i=1, \ldots, r-1$.

Clearly, this subsequence has the properties $t(1)<t(2)<\ldots<t(r)$ and $f_{t(1)}>f_{t(2)}>\ldots>f_{t(r)}$. Moreover, $f_{t(r)}=m_{k}$. Hence, given the subsequence, the desired minimum is immediately available.

We keep track of the subsequence by storing its elements in decreasing order in a list (i.e, $f_{k+1}$ is the element at the top). This particular data structure has the property that elements at the bottom can only be deleted, while elements at the top can be both deleted and added. Hence, it could 
be viewed as a combination of a stack and a queue, and it can easily be implemented so that each deletion and each addition requires constant time (see [2]).

To see why this data structure is convenient, first observe the following: if for a given $k \geq 2$, a value $l \in\left\{k+1, k+2, \ldots, u_{k}\right\}$ is not selected in the subsequence, then $l$ will not be selected for $k-1$. This follows from the fact that there exists some value in the subsequence, say $t(i)$, such that $k+1 \leq t(i)<l$ and $f_{t(i)}<f_{l}$. Now if $l \in\left\{k, k+1, \ldots, u_{k-1}\right\}$, then also $t(i) \in\left\{k, k+1, \ldots, u_{k-1}\right\}$ and this implies that $l$ should still be excluded from the subsequence. Repetition of this argument leads to an element never being considered for inclusion in the subsequence once it has been excluded. This means that when for a certain $k \geq 2$ the elements $t(1), t(2), \ldots, t(r)$ of the subsequence are given, then - once $u_{k-1}$ is known - the corresponding

subsequence for $k-1$ can be constructed as follows. Because $u_{k-1} \leq u_{k}$, we first delete from the bottom of the list any element larger than $u_{k-1}$. Now, suppose $u_{k-1}>k-1$. Then, because $k$ will be added at the top of the list, we delete from the top all remaining elements $t(i)$ for which $f_{k} \leq f_{t(i)}$. Finally, we add $k$ at the top of the list. In case $u_{k-1}=k-1$ the list is empty after the deletion operations and no element is added.

The above updating process is carried out $n-1$ times in total. Each time at most one element is added, which requires constant time per addition. Furthermore, several elements may be deleted. Note that, because the list elements are already ordered, each deletion requires indeed constant time. The number of deleted elements can not be bounded nicely for each individual time the updating process is carried out. However, the overall number of deletions is not larger than $n$. The reason for this is simple: in the updating process, each of the elements $1,2, \ldots, n$ is added at most once to the list and therefore it can be deleted at most once.

To summarize the above discussion: we have shown that problem $(\mathrm{P})$ can be solved in $O(n)$ time.

\section{Scheduling jobs of a single type under batch availability}

The problem we are addressing in this section may be stated formally as follows. There are $n$ jobs to be scheduled on a single machine. Each job $j(j=1, \ldots, n)$ has a processing time $p_{j}$ and a due date $d_{j}$ by which it should ideally complete. Jobs can be processed consecutively in batches. At the start of the schedule and prior to each batch, a set-up time $s$ is 
incurred, which motivates the formation of longer batches so as to reduce the completion time of later jobs. However, batch availability applies, which means that all the jobs that belong to the same batch complete only when the last job in the batch completes. As a consequence, extending a batch by including additional jobs increases the completion time of the jobs previously in the batch.

The above problem setting is introduced in [12]. For the sum of completion times objective, an efficient algorithm is given by Coffman et al. [7]: the batching step is performed in linear time to give an overall time requirement of $O(n \log n)$. An extension of this algorithm for a slightly more general cost function is proposed by Albers and Brucker [3] (see also [5]). It is worth pointing out that the approach in [7,3], like ours, relies on the notion of a queue. However, in the problem examined here, the presence of a maximum operation within the dynamic programming recursion is an additional complication that does not arise in the sum of completion times variant. (This is also true for the problems addressed in later sections.)

It is shown in [15] that there is an optimal schedule in which jobs complete according to the earliest due date (EDD) rule. Thus, the jobs can be re-indexed according to this rule in $O(n \log n)$ time and the problem reduces to one of batching that can be solved using a backward dynamic program with batch insertion. Let $G_{k}$ denote the minimum overall lateness of a schedule containing jobs $k, k+1, \ldots, n$ when starting at time 0 . The initialization is $G_{n+1}=-\infty$ and the recursion for $k=n, n-1, \ldots, 1$ is

$$
G_{k}=\min _{k<l \leq n+1}\left\{\max \left\{\left(s+a_{k}-a_{l}\right)+G_{l},\left(s+a_{k}-a_{l}\right)-d_{k}\right\}\right\},
$$

where $a_{k}=\sum_{h=k}^{n} p_{h}$ for $k=1, \ldots, n$. Here $l$ denotes the first job in the second batch of the schedule. Since this batch starts at time $s+a_{k}-a_{l}$, the minimum overall lateness from this batch onward is given by the first term between brackets, while the lateness of the first batch is given by the other term (since job $k$ has the smallest due date).

As pointed out in [15], a straightforward implementation of the above algorithm requires $O\left(n^{2}\right)$ time. However, we now show that the dynamic programming part can be implemented in linear time.

From (3), or common sense reasoning, it follows that $G_{l+1} \leq G_{l}$ for every $l \leq n-1$. Hence, if $G_{l+1} \geq-d_{k}$ for some $k \in\{1, \ldots, n\}$, then also $G_{l} \geq-d_{k}$. We now define $q_{k}$ as the largest job index $l$ in $\{k+1, \ldots, n\}$ such that $G_{l} \geq-d_{k}$; if no such index exists, we define $q_{k}=k$. (Note that, because of the EDD order, $q_{k+1} \geq q_{k}$ holds for every $k \leq n-1$.)

From the above observations, it follows that for all indices $l \in I_{k}^{1}=$ 
$\left\{k+1, \ldots, q_{k}\right\}$ the maximum in (3) is given by the first term, whereas for $l \in I_{k}^{2}=\left\{q_{k}+1, \ldots, n+1\right\}$, the maximum is given by the second term. Now (3) can be rewritten as

$$
G_{k}=\min \left\{\min _{k<l \leq q_{k}}\left\{s+a_{k}-a_{l}+G_{l}\right\}, \min _{q_{k}<l \leq n+1}\left\{s+a_{k}-a_{l}-d_{k}\right\}\right\} .
$$

Note that, for this problem, each of $I_{k}^{1}$ and $I_{k}^{2}$ is contiguous. Further, the second minimum is always attained for $l=q_{k}+1$ : owing to the batch availability assumption and the EDD indexing of the jobs, the overall lateness of a batch is always determined by the first job in the batch. Consequently, the remaining task is to compute

$$
s+a_{k}+\min _{k<l \leq q_{k}}\left\{-a_{l}+G_{l}\right\}
$$

efficiently. However, since this has to be done for every value of $k$, we actually need to solve an instance of problem (P) with $u_{k}=q_{k}$ and $f_{l}=-a_{l}+G_{l}$. Hence, it takes overall $O(n)$ time to calculate the minima given by (4).

Since the parameters $a_{l}, l=1,2, \ldots, n$, and, because of monotonicity, the values $q_{k}, k=1,2, \ldots, n$, can be computed in $O(n)$ time, we have now shown that the time requirement of our algorithm to solve the batching problem is linear. Hence, because of the sorting step, the overall time requirement is $O(n \log n)$. This constitutes an improvement over the algorithm in $[15]$.

\section{Scheduling jobs on a batching machine}

The problem we are addressing in this section may be stated formally as follows. There are $n$ jobs to be processed on a single batching machine. This machine is capable of processing up to $b$ jobs simultaneously in batches. Each job $j(j=1, \ldots, n)$ has a processing time $p_{j}$ and a due date $d_{j}$ by which it should ideally complete. Whenever a batch is formed, its completion time is equal to the largest processing time of any job in the batch.

The model is analyzed extensively in a recent paper by Brucker et al. [6]. They distinguish between the unbounded case where $b \geq n$ and the bounded case whereby $b<n$. For the unbounded problem of minimizing the maximum lateness, it is shown in [6] that there is an SPT-batch optimal schedule. Thus, the jobs can be re-indexed according to this rule in $O(n \log n)$ time and the problem reduces to one of batching that can be solved using the following backward dynamic program with batch insertion of Brucker et al. [6]. Let $G_{k}$ denote the minimum overall lateness of a schedule containing 
jobs $k, k+1, \ldots, n$ when starting at time 0 . The initialization is $G_{n+1}=-\infty$ and the recursion for $k=n, n-1, \ldots, 1$ is

$$
G_{k}=\min _{k<l \leq n+1}\left\{\max \left\{p_{l-1}+G_{l}, p_{l-1}+\max _{k \leq j \leq l-1}\left\{-d_{j}\right\}\right\}\right\},
$$

where $l$ should again be interpreted as the first job of the second batch, which starts when the first batch completes. By definition, this happens when the longest job $(l-1)$ of the first batch completes.

A standard implementation of the above algorithm, as proposed in [6], requires $O\left(n^{2}\right)$ time. We now show that the dynamic programming part can be implemented in linear time, thus yielding an overall time requirement of $O(n \log n)$.

Our approach is somewhat similar to the one in the previous section. Again it can easily be verified that $G_{l+1} \leq G_{l}$ for every $l \leq n-1$. Hence, if $G_{l+1}>\max _{k<j \leq l}\left\{-d_{j}\right\}$ for some $k \in\{1, \ldots, n\}$, then

$$
G_{l} \geq G_{l+1}>\max _{k<j \leq l}\left\{-d_{j}\right\} \geq \max _{k<j \leq l-1}\left\{-d_{j}\right\} .
$$

It follows that, if $I_{k}^{1}$ and $I_{k}^{2}$ are defined as in Section 2 (that is: $I_{k}^{1}=\{l \in$ $\left.\{k+1, \ldots, n\} \mid G_{l} \geq \max _{k<j \leq l-1}\left\{-d_{j}\right\}\right\}$ and $\left.I_{k}^{2}=I_{k}^{1} \cap\{k+1, \ldots, n+1\}\right)$, then, $I_{k}^{1}=\left\{k+1, \ldots, q_{k}\right\}$ and $I_{k}^{2}=\left\{q_{k}+1, \ldots, n+1\right\}$, where $q_{k}$ is the largest index with the required property. For convenience we define $q_{k}=k$ if the inequality is not satisfied by any job in $\{k+1, k+2, \ldots, n\}$. Note that $q_{k}$ is non-decreasing in $k$. Recursion formula (5) can now be rewritten as

$$
G_{k}=\min \left\{\min _{k<l \leq q_{k}}\left\{p_{l-1}+G_{l}\right\}, \min _{q_{k}<l \leq n+1}\left\{p_{l-1}+\max _{k \leq j \leq l-1}\left\{-d_{j}\right\}\right\}\right\} .
$$

The first minimization problem between brackets can again be viewed as an instance of problem (P) with $u_{k}=q_{k}$ and $f_{l}=p_{l-1}+G_{l}$. With respect to the second minimization problem, we observe that, for a fixed arbitrary $k$, the minimum is attained for $l$ as small as possible, i.e. $l=q_{k}+1$, since this minimizes both the term $p_{l-1}$, because of the SPT order, as well as the range over which the maximum is computed. Hence, we are left with calculating

$$
p_{q_{k}}+\max _{k \leq j \leq q_{k}}\left\{-d_{j}\right\}=p_{q_{k}}+\max \left\{-d_{k}, \max _{k<j \leq q_{k}}\left\{-d_{j}\right\}\right\} .
$$

This boils down to solving the problem

$$
\min _{k<j \leq q_{k}}\left\{d_{j}\right\}
$$


which is an instance of $(\mathrm{P})$ with $u_{k}=q_{k}$ and $f_{j}=d_{j}$. From these observations and the fact that, because of monotonicity, the values $q_{k}, k=$ $1,2, \ldots, n$, can be computed in $O(n)$ time, it follows that the time requirement of our algorithm to solve the batching problem is linear. Hence, taking into account the SPT-sorting step, the overall time requirement is again $O(n \log n)$. This constitutes an improvement over the algorithm in [6].

Finally, we note that Brucker et al. [6] use their algorithm for minimizing the maximum lateness as a subroutine in a polynomial procedure for minimizing the maximum cost. Therefore, the $O(\log n)$ improvement obtained here applies to that procedure too.

\section{$5 \quad$ Scheduling customized two-operation jobs}

The problem we are addressing in this section may be stated formally as follows. There are $n$ jobs which have to be scheduled on a single machine. Each job $j(j=1,2, \ldots, n)$ has two operations, namely a standard operation followed - not necessarily immediately - by a specific operation. These operations have processing times $p_{j}^{(1)}$ and $p_{j}^{(2)}$, respectively. A set-up time is required before the first standard operation and whenever there is a switch in production from specific to standard operations; two standard operations may be processed consecutively to form a batch without a set-up in between. With respect to the way in which standard operations are released (become available) after processing, two schemes are possible: batch availability, defined in Section 3, and the alternative item availability whereby an operation becomes available immediately after it has been processed. We only analyze the batch availability variant explicitly and give comments as to how the result can be extended to the item availability case.

The model is introduced in [4] (for batch availability) and then analyzed for due-date related criteria in [10]. We note that the problem discussed in [4] for the sum of completion times objective was shown to be equivalent to the, seemingly simpler, problem studied in [7]. In particular, it was shown that the specific (unique) operations can essentially be removed from the problem. If this were also the case for the maximum lateness variants of these problems, then the results of Section 3 could be used directly to solve the problem discussed in this section. Before we proceed with our analysis, it is worthwhile to show that this is not the case. Consider the instance of the two-operation variant in which the set up time is $c(c>0)$ and there are three jobs with due dates and operation processing times as shown in Table 1. 
Table 1: Job data 1

\begin{tabular}{l|ccc}
\hline Job $i$ & 1 & 2 & 3 \\
\hline$p_{i}^{(1)}$ & 1 & 1 & 1 \\
$p_{i}^{(2)}$ & 1 & 1 & $c+2$ \\
\hline$d_{j}$ & $c+1$ & $2 c+3$ & $2 c+3$ \\
\hline
\end{tabular}

It can be easily verified that the problem of Section 3 obtained by omitting the specific operations, has as the unique optimal solution job 1 in the first batch and jobs 2 and 3 in the second batch. The value of this solution is $L_{\max }=0$. However, inserting the specific operations into this schedule (immediately after the corresponding batch) yields a schedule for the two-component problem with lateness equal to $c+4$. It is easy to see that scheduling all the standard operations in one batch, followed by all the specific operations in EDD order, yields a schedule with lateness of 4. Thus, our example suggests that there is no obvious way to translate optimal solutions to the problem in Section 3 into optimal solutions for the problem in this section. This observation and the analysis below seem to lead to the conclusion that the problem in this section is genuinely more complex.

Returning to the two-operation problem, it is shown in [10] that there is an optimal schedule in which jobs complete according to the EDD rule. Thus, the jobs can be re-indexed according to this rule in $O(n \log n)$ time and the problem reduces to one of batching that can be solved using a backward dynamic program with batch insertion [10]. Let $G_{k}$ denote the minimum overall lateness of a schedule containing jobs $k, k+1, \ldots, n$. The initialization is $G_{n+1}=-\infty$ and the recursion for $k=n, n-1, \ldots, 1$ is

$$
G_{k}=\min _{k<l \leq n+1}\left\{\max \left\{\begin{array}{l}
s+\sum_{h=k}^{l-1} p_{h}^{(1)}+\sum_{h=k}^{l-1} p_{h}^{(2)}+G_{l}, \\
s+\sum_{h=k}^{l-1} p_{h}^{(1)}+\max _{k \leq j \leq l-1}\left\{\sum_{h=k}^{j} p_{h}^{(2)}-d_{j}\right\}
\end{array}\right\}\right\} .
$$

Again, $G_{k+1} \leq G_{k}$ for $k=1,2, \ldots, n-1$ holds. A standard implementation of the above algorithm requires $O\left(n^{2}\right)$ time, if some preprocessing is used. We now show that the dynamic programming part can be implemented in $O(n \log n)$ time thus yielding an overall time requirement of $O(n \log n)$.

For the maximum in (6) to be given by the first term, the following needs 
to hold

$$
G_{l} \geq \max _{k<j \leq l-1}\left\{-\sum_{h=j+1}^{l-1} p_{h}^{(2)}-d_{j}\right\}
$$

or equivalently

$$
G_{l}-\sum_{h=l}^{n} p_{h}^{(2)} \geq \max _{k \leq j \leq l-1}\left\{-\sum_{h=j+1}^{n} p_{h}^{(2)}-d_{j}\right\} .
$$

Consider an arbitrary index $k \in\{2,3, \ldots, n\}$. Let the subset $I_{1}^{k} \subseteq$ $\{k+1, k+2, \ldots, n\}$ contain the indices for which (7) holds. We first explain how we determine $I_{1}^{k-1}$. Since the left-hand-side value of (7) does not depend on $k$ and

$$
\max _{k \leq j \leq l-1}\left\{-\sum_{h=j+1}^{n} p_{h}^{(2)}-d_{j}\right\} \leq \max _{k-1 \leq j \leq l-1}\left\{-\sum_{h=j+1}^{n} p_{h}^{(2)}-d_{j}\right\}
$$

it holds that $\left(I_{1}^{k-1} \cap\{k+1, k+2, \ldots, n\}\right) \subseteq I_{1}^{k}$. Moreover, the elements of $I_{1}^{k}$ which are not in $I_{1}^{k-1}$ are exactly those $l \in I_{1}^{k}$ for which

$$
G_{l}-\sum_{h=l}^{n} p_{h}^{(2)}<-\sum_{h=k}^{n} p_{h}^{(2)}-d_{k-1} .
$$

Note that the right-hand-side of (8) is a constant for fixed $k$. Hence, if the inequality is satisfied for one or more indices in $I_{1}^{k}$, then these correspond to the smallest elements of the set $\left\{G_{l}-\sum_{h=l}^{n} p_{h}^{(2)} \mid l \in I_{1}^{k}\right\}$. This fact can be used to efficiently determine $I_{1}^{k-1}$. In our implementation, we make use of a heap, which we denote by $H_{1}$ Recall that this data structure has the following properties [2]:

(i) the minimum of all values stored in the heap can be retrieved in constant time,

(ii) adding a value to the heap takes $O(\log m)$ time, where $m$ is the number of stored values,

(iii) deleting a value from the heap takes $O(\log m)$ time.

Suppose that heap $H_{1}$ contains the values $G_{l}-\sum_{h=l}^{n} p_{h}^{(2)}$ for all $l \in I_{1}^{k}$. After $G_{k}$ has been calculated (how this is done efficiently will be shown below), we would like $H_{1}$ to contain the values $G_{l}-\sum_{h=l}^{n} p_{h}^{(2)}$ for all $\left.l \in I_{1}^{k-1}\right\}$. To achieve this, we first check whether the minimum value is less than the 
right-hand-side of (8). If this is the case, then we delete the minimum from $H_{1}$ and we repeat the comparison with the new minimum value. We keep deleting the current minimum value from $H_{1}$ until this value becomes at least the as large as the right-hand-side of (8) or until $H_{1}$ is empty. Then we check whether $G_{k}-\sum_{h=k}^{n} p_{h}^{(2)}$ is at least as large as the right-hand-side of (8). Only if this is the case, do we add $G_{k}-\sum_{h=k}^{n} p_{h}^{(2)}$ to $H_{1}$. At this point, $H_{1}$ contains the values $G_{l}-\sum_{h=l}^{n} p_{h}^{(2)}$ for all $l \in I_{1}^{k-1}$. In parallel to updating $H_{1}$, we can keep track of the indices that correspond to its elements.

Let us now turn to the issue of the efficient calculation of $G_{k}$. From the definition of $I_{1}^{k}$ it follows that we would like to calculate

$$
s+\min _{l \in I_{1}^{k}}\left\{\sum_{h=k}^{l-1} p_{h}^{(1)}+\sum_{h=k}^{l-1} p_{h}^{(2)}+G_{l}\right\}
$$

and

$$
s+\min _{l \in I_{2}^{k}} \max _{k \leq j \leq l-1}\left\{\sum_{h=k}^{l-1} p_{h}^{(1)}+\sum_{h=k}^{j} p_{h}^{(2)}-d_{j}\right\},
$$

where $I_{2}^{k}=\{k+1, k+2, \ldots, n+1\} \backslash I_{1}^{k}$.

First consider (10). Suppose $l, i \in I_{2}^{k}$ and $l<i$, then

$$
\begin{aligned}
& \max _{k \leq j \leq l-1}\left\{\sum_{h=k}^{l-1} p_{h}^{(1)}+\sum_{h=k}^{j} p_{h}^{(2)}-d_{j}\right\} \leq \\
& \max _{k \leq j \leq l-1}\left\{\sum_{h=k}^{i-1} p_{h}^{(1)}+\sum_{h=k}^{j} p_{h}^{(2)}-d_{j}\right\} \leq \\
& \max _{k \leq j \leq i-1}\left\{\sum_{h=k}^{i-1} p_{h}^{(1)}+\sum_{h=k}^{j} p_{h}^{(2)}-d_{j}\right\} .
\end{aligned}
$$

It follows that the minimum in (10) is attained for the smallest element of $I_{2}^{k}$, which we denote by $q_{k}$; we define $q_{k}=k$ if $I_{2}=\emptyset$. Hence, (10) is equivalent to

$$
s+\max _{k \leq j \leq q_{k}-1}\left\{\sum_{h=k}^{q_{k}-1} p_{h}^{(1)}+\sum_{h=k}^{j} p_{h}^{(2)}-d_{j}\right\}
$$

or

$$
s-\sum_{h=k}^{q_{k}-1} p_{h}^{(1)}-\sum_{h=1}^{k-1} p_{h}^{(2)}+\max \left\{\sum_{h=1}^{k} p_{h}^{(2)}+d_{k},-\min _{k<j \leq q_{k}-1}\left\{-\sum_{h=1}^{j} p_{h}^{(2)}+d_{j}\right\}\right\} .
$$


From the discussion about the updating process of heap $H_{1}$, it follows that the values $q_{k}$ are non-decreasing in $k$. (Also note that keeping track of the values $q_{k}, k=1,2, \ldots, n$, requires overall $O(n)$ time.) Hence, the minimization is an instance of $(\mathrm{P})$ with $u_{k}=q_{k}-1$ and $f_{j}=-\sum_{h=1}^{j} p_{h}^{(2)}+d_{j}$. It follows that (10) can be calculated for all values of $k=1,2, \ldots, n$ together in linear time.

For the efficient calculation of (9), we use a heap $H_{2}$ which contains the values $\sum_{h=1}^{l-1} p_{h}^{(1)}+\sum_{h=1}^{l-1} p_{h}^{(2)}+G_{l}$ for all $l \in I_{1}^{k}$ and possibly for some $l \in I_{2}^{k}$. Note that these values are independent of $k$. To calculate (9), we simply retrieve the minimum from the heap. If the minimum corresponds to an element of $I_{2}^{k}$, we delete this value from $\mathrm{H}_{2}$ and retrieve the new minimum. This is repeated until the minimum corresponds to an element of $I_{1}^{k}$ or until $H_{2}$ is empty. In the latter case the value of (9) is $\infty$, while in the former case we get the value of (9) by adding $s$ and subtracting $\sum_{h=1}^{k-1} p_{h}^{(1)}+\sum_{h=1}^{k-1} p_{h}^{(2)}$.

The time complexity of the above algorithm depends on the number of additions to and deletions from the heaps. For every $l=1,2, \ldots, n$, the value $G_{l}-\sum_{h=l}^{n} p_{h}^{(2)}$ is added at most once to $H_{1}$ and the value $\sum_{h=1}^{l-1} p_{h}^{(1)}+$ $\sum_{h=1}^{l-1} p_{h}^{(2)}+G_{l}$ is added at most once to $H_{2}$. (These additions actually occur at the same point in time.) Furthermore, deletion from $H_{1}$ and $H_{2}$ also occurs at most once for every index. Since the heaps never contain more than $n$ elements, it follows that the total computational effort involving heap operations is $O(n \log n)$.

Finally, we note that partial sums such as $\sum_{h=k}^{l-1} p_{h}^{(1)}$ can be replaced by $\sum_{h=k}^{n} p_{h}^{(1)}-\sum_{h=l}^{n} p_{h}^{(1)}$. Partial sums of the latter type can be calculated in linear time in a preprocessing step.

We have now arrived at the required result: our algorithm solves the batching problem in $O(n \log n)$ time thus yielding an overall time requirement of $O(n \log n)$ time. This constitutes an improvement over the algorithm in $[10]$.

With respect to the item availability case, we note that the problem can be solved using a double recursion dynamic program with block insertion; such a scheme is proposed in [9] and enables us to deploy the approach developed in this section 'twice' (in parallel, even) to reduce the overall complexity to $O(n \log n)$. 


\section{Concluding Remarks}

We have presented improved dynamic programming algorithms for a class of scheduling problems involving the maximum lateness criterion and an element of batching. A question that arises is whether insights gained from this study can help to reduce the time requirement of algorithms for other, more complicated, models involving the batching of jobs that belong to different families. Since, in that context, the tasks of sequencing and batching can only be separated within each family but not at the overall level, it is not obvious whether or how our approach could be applied to those problems.

An interesting research direction is that of ascertaining whether there exist maximization problems outside the domain of production scheduling that are amenable to our approach.

\section{Acknowledgements}

The authors wish to thank Chris Potts who suggested this research topic. Financial support by the Tinbergen Institute is gratefully acknowledged.

\section{References}

[1] A Aggarwal and J K Park. Improved algorithms for economic lot size problems. Operations Research, 41(3):549-571, 1993.

[2] A V Aho, J E Hopcroft, and J D Ullman. Data Structures and Algorithms. Addison-Wesley, Reading, MA, 1987.

[3] S Albers and P Brucker. The complexity of one-machine batching problems. Discrete Applied Mathematics, 47:87-107, 1993.

[4] K R Baker. Scheduling the production of components at a common facility. IIE Transactions, 20(1):32-35, 1988.

[5] P Brucker. Scheduling Algorithms. Springer Verlag, Berlin, 1995.

[6] P Brucker, A Gladky, H Hoogeveen, M Y Kovalyov, C N Potts, T Tautenhahn, and S van de Velde. Scheduling a batching machine. Journal of Scheduling, 1:31-54, 1998.

[7] E G Coffman, M Yannakakis, M J Magazine, and C Santos. Batch sizing and job sequencing on a single machine. Annals of Operations Research, 26:135-147, 1990. 
[8] A Federgruen and M Tzur. A simple forward algorithm to solve general dynamic lot sizing models with $n$ periods in $O(n \log n)$ or $O(n)$ time. Management Science, 37:909-925, 1991.

[9] A E Gerodimos, C A Glass, and C N Potts. Scheduling customised jobs on a single machine under item availability. submitted to IIE Transactions in Operations Research, 1998.

[10] A E Gerodimos, C A Glass, and C N Potts. Scheduling the production of two-operation jobs on a single machine. to appear in a Feature Issue of European Journal of Operational Research dedicated to the 15th EURO Summer Institute, ESI XV, on Production Scheduling, St Vincent, Aosta Valley, Italy, September 12-26, 1997, 1998.

[11] M Y Kovalyov and C N Potts. Scheduling with batching: A review. to appear in a Feature Issue of European Journal of Operational Research dedicated to the 15th EURO Summer Institute, ESI XV, on Production Scheduling, St Vincent, Aosta Valley, Italy, September 12-26, 1997, 1998.

[12] C A Santos and M J Magazine. Batching in single operation manufacturing systems. Operations Research Letters, 4(3):99-103, 1985.

[13] S van Hoesel, A Wagelmans, and B Moerman. Using geometric techniques to improve dynamic programming algorithms for the economic lot-sizing problem and extensions. European Journal of Operational Research, 75:312-331, 1994.

[14] A Wagelmans, S van Hoesel, and A Kolen. Economic lot sizing: An $O(n \log n)$ algorithm that runs in linear time in the Wagner-Whitin case. Operations Research, 40(Supp. 1):145-156, 1992.

[15] S Webster and K R Baker. Scheduling groups of jobs on a single machine. Operations Research, 43:692-703, 1995. 